\title{
Template für eine akademische Karriere in der Hausarztmedizin
}

\author{
Ryan Tandjung ${ }^{a}$, Andreas Zeller ${ }^{b}$, Peter Jüni ${ }^{c}$, Thomas Rosemann ${ }^{a}$, Thomas Bischoff ${ }^{d}$, Johanna Sommer ${ }^{e}$ \\ ${ }^{a}$ Institut für Hausarztmedizin Zürich; ${ }^{b}$ Universitäres Zentrum für Hausarztmedizin beider Basel; ${ }^{c}$ Berner Institut für Hausarztmedizin; \\ dInstitut Universitaire de Médecine Génerale, Lausanne; e Unité de Médecine de Premiers Recours, Genève
}

Die universitären Institute für Hausarztmedizin sind die akademische Heimat der Hausärztinnen und Hausärzte, die sich in Lehre und Forschung engagieren. Sie stellen mit ihrer Infrastruktur das Rückgrat und die Kontinuität sicher, die für eine starke Position in Klinik, Lehre und Forschung zentral ist.

Die Institute für Hausarztmedizin zeichnen sich aus durch ihre Aktivitäten zur Förderung und Stärkung der Akademisierung der Hausarztmedizin mit den untrennbaren Gebieten der Klinik, Forschung und Lehre:

\section{Die klinische Tätigkeit}

Die Aufgabe der Institute ist es, eine hohe Qualität in der klinischen Tätigkeit der Hausarztmedizin zu gewährleisten. In diesem Sinne erarbeiten die Institute für Hausarztmedizin Richtlinien für eine evidenzbasierte und effiziente Hausarztmedizin, die der spezifischen Population in der Grundversorgung entspricht. Eine klinische Tätigkeit der akademisch tätigen Hausärztinnen und Hausärzte stellt die Regel dar. Die klinische Tätigkeit gewährleistet die Verbindung zum Patienten und zur Basis in der Hausarztmedizin.

\section{Die Forschung}

Die Forschungstätigkeit an den Instituten für Hausarztmedizin ist von grosser Bedeutung. Die akademischen Hausärztinnen und Hausärzte forschen auf internationalem Niveau und verschaffen dem Fach damit die Anerkennung in der Fakultät. Das Ziel hausärztlicher Forschung ist die Verbesserung der Versorgung von Patientinnen und Patienten in der Hausarztpraxis, sie dient darüber hinaus auch als Basis für die Lehre. Die Infrastruktur sichert einen niederschwelligen Zugang zu methodischen Kompetenzen z.B. in Statistik oder Epidemiologie. Dies sichert einerseits die Konstanz bei Projekten und die Vermittlung von Methodenkompetenz an die forschenden Hausärztinnen und Hausärzten. Die hausärztliche Forschung strebt eine enge Zusammenarbeit unter den Instituten für Hausarztmedizin und mit verschiedenen weiteren Fachdisziplinen an.

PD Dr. med. Ryan Tandjung Institut für Hausarztmedizin UniversitätsSpital Zürich Pestalozzistrasse 24 CH-8091 Zürich ryan.tandjung[at]usz.ch
Evidenz. Die Institute für Hausarztmedizin setzen sich dafür ein, dass die Didaktik in den Vorlesungen, Seminaren und insbesondere im Einzeltutoriat in der Hausarztpraxis den Qualitätskriterien der medizinischen Lehre entspricht. Dem Kontakt mit dem Patienten kommt eine zentrale Bedeutung zu. Mit der didaktischen Forschung in der Lehre fördern die Institute für Hausarztmedizin eine bedeutende Entwicklung der Lehrkompetenzen in ihrem Gebiet.

In der Lehre tätige Hausärztinnen und Hausärzte sollen in die Gestaltung des Curriculums einbezogen werden, um hausärztliche Lehrveranstaltungen in den einzelnen Lerneinheiten angemessen zu berücksichtigen.

\section{Karriereförderung}

Künftige akademische Hausärztinnen und Hausärzte sollen bei den Assistentinnen und Assistenten in der Weiterbildung zur Allgemeinen Inneren Medizin (zum Beispiel während der Weiterbildung in Universitätskliniken) rekrutiert werden. Ihnen soll der Weg einer Habilitation aufgezeigt werden; eine Beteiligung in allen drei Bereichen (Klinik, Forschung und Lehre) mit unterschiedlichen Schwerpunkten ist anzustreben.

\section{Weiter- und Fortbildung}

Die Institute für Hausarztmedizin nehmen in der Weiterund Fortbildung eine wichtige Rolle ein. In der Weiterbildung beteiligen sie sich an der Koordination und Qualitätssicherung von Praxisassistenzprogrammen und Rotationsstellen/Curricula. In der Fortbildung publizieren sie auf der Basis der eigenen Forschung und verfügbaren Literatur Stellungnahmen und Guidelines für die tägliche Versorgung der Patienten. Bei Fortbildungsveranstaltungen nehmen sie eine führende Position ein, um diese Richtlinien, die eigenen hausärztlichen Fortbildungsinhalte und insbesondere auch Ergebnisse hausärztlicher Forschungstätigkeit an die Schweizer Hausärzte zu vermitteln.

\section{Ergänzung}

Diese Stellungnahme erfolgt im Rahmen der Swiss Academy of Family Medicine (Safmed), der Kooperation der fünf universitären Institute für Hausarztmedizin. Herzlichen Dank an Adrian Göldlin (Bern), Lilli Herzig (Lausanne), Dagmar Haller-Hester (Genf) und Peter Tschudi (Basel) für ihren Beitrag zu dieser Stellungnahme. 